\title{
ABUNDANCE EFFECTS IN THE CLASSIFICATION OF K STARS
}

\author{
P. M. WILLIAMS
}

Royal Observatory, Edinburgh, United Kingdom

\begin{abstract}
The influence of metal abundance and gravity on the relation between spectral type and effective temperatures of late $\mathbf{G}$ and $\mathrm{K}$ type stars is investigated and calibrated using metal abundances from narrow-band photometry, near infrared photometry and independent luminosity estimates.
\end{abstract}

\section{Introduction}

While spectral classification of the coolest and most highly evolved stars has always reflected their composition owing to the conspicuous effects on their spectra of the carbon-to-oxygen abundance ratio, the effects of the overall metal abundance on stars' spectra are more subtle. They are also more liable to misinterpretation among those spectral types dependent on metal to hydrogen line ratios and the present contribution is an attempt to calibrate such affects among the $\mathrm{G}$ and $\mathrm{K}$ type giants. In principle, there are two ways of going about this - either by simulating the classification criteria using synthetic spectra and finding the model atmosphere parameters corresponding to the class boundaries, or by using the stars themselves to investigate the transformation from spectral classifications to the $[T, g, x]$ (Temperature, gravity, metal abundance) space. As the first approach also involves simulation of the application of the classification criteria, the second approach using stars already classified by experienced observers is used here. The $\mathrm{G}$ and $\mathrm{K}$ type stars cover wide ranges of mass, age and metal abundance and have also been the subjects of numerous abundance analyses, making them very suitable for such a study. It is appropriate at this meeting to recall that nearly 40 years ago, Dr. W. W. Morgan, in a paper (Morgan, 1937) foreshadowing the two-dimensional spectral classification, emphasised the fundamental nature of the effective temperature - surface gravity diagram and its advantages over the HR diagram. The fundamental nature of surface gravity was noted by Pannekoek (1922) who wrote '.... the physical quantity, directly given by the spectra used for the determination of spectroscopic parallaxes, is the gravitation at the surface of the star.' Inevitably, the determination of spectroscopic absolute magnitudes and the calibration of luminosity classifications in terms of absolute magnitude must also involve knowledge of the gravity-luminosity relation which itself requires a mass-luminosity relation. Even if these are known, it can be shown (e.g. Williams, 1971) that a relative error of a factor of two in the gravity, which is by no means pessimistic even for a high dispersion spectroscopic analysis, corresponds to one magnitude in $M_{\nu}$. Therefore gravity is preferable to luminosity or absolute magnitude for the second axis of calibration. 


\section{Calibration}

The stars used for this calibration comprise a sample of G8 to K4 giants, omitting known spectroscopic binaries and stars with 'peculiar' classifications, with spectral classifications by Johnson and Morgan (1953) or Roman (1952) and for which metal abundances were derived in a narrow-band photometric programme. Since this has already been described elsewhere (Williams, 1971, 1972), it is sufficient here to recall that the metal abundances were derived from spectrophotometric indices analysed by spectral syntheses and are accurate to within 0.2 in $[\mathrm{Fe} / \mathrm{H}]$ and that the temperatures, determined primarily from $(I-K)$ photometry calibrated with model atmospheres and empirically calibrated $(R-I)$, are essentially independent of metal abundance. The temperatures are effectively model atmosphere labels rather than true effective temperatures which cannot be determined experimentally for any but a small number of stars. The gravities were determined from $M_{v}(K)$ with an abundance term, trigonometric and dynamical parallaxes and theoretical mass-luminosity relations. They are probably accurate to within a factor of two and no calibration of luminosity class against gravity is attempted here.

First, consider the disposition of stars of a given spectral type in $[T, g, x]$ space. This is illustrated in Figure 1 for the K0 stars, which comprise the most populous class. The stars were divided into ranges of width $\Delta[\mathrm{Fe} / \mathrm{H}]=0.2$ and $\Delta \log g=0.3$ and the average

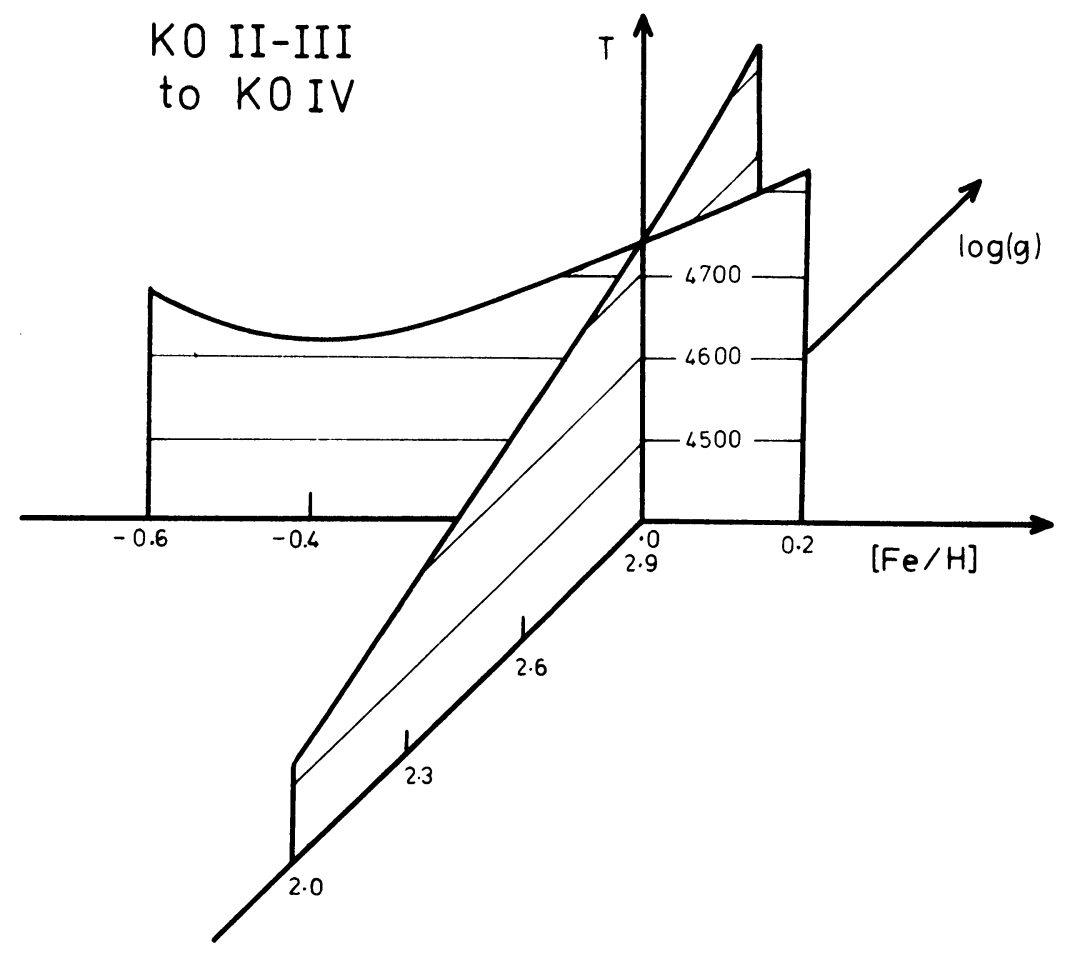

Fig. 1. Disposition of $\mathrm{K} 0$ giants and subgiants in temperature-gravity-metal abundance space. 
temperature found for the stars in each. Owing to the paucity of stars in much of the field, only those in the gravity range $2.8 \leqslant \log g<3.1$ were plotted against metal abundance, and stars having $-0.3<[\mathrm{Fe} / \mathrm{H}]<0.3$ were combined for the gravity axis. The features are as one might expect; the only surprise being the turn up in temperature at the low abundance end. This is also observed among the $\mathrm{K} 3$ stars, although there are so few of these to make the result even more uncertain, and the G8 stars. Taken together, these results suggest that it is mainly among the mildly metal poor stars, with $[\mathrm{Fe} / \mathrm{H}] \sim-0.5$, that the classification is most affected by metal abundance. The magnitude of the effect is about $100 \mathrm{~K}$ or $1-2$ sub-types in spectral class. The dispersion in temperature of stars in any one range is usually between $50 \mathrm{~K}$ and $100 \mathrm{~K}$, so that even where the metal abundance and gravity are known, the spectral type is barely a good measure of the temperature. Among stars of later types, the dispersions are generally less.

Secondly, for an overall idea of the sensitivity of the relation between spectral type and temperature, regression lines were fitted to the $T-\log g$ and $T-[\mathrm{Fe} / \mathrm{H}]$ data for stars of each type, limiting the abundances in the first case to $-0.2<[\mathrm{Fe} / \mathrm{H}]<0.2$ and the gravities in the second by considering stars of luminosity class III only. The slopes of these lines are given in Table I together with the differences $\Delta \log g$ and $\Delta[\mathrm{Fe} / \mathrm{H}]$ which will affect the spectral classification by one sub type. As there were so few $\mathrm{K} 1$ and $\mathrm{K} 4$ type stars, the results for these were combined with those for the K2 and K3 stars in the table, although the slopes were, of course, determined separately.

TABLE I

Abundance and gravity effects on classification

\begin{tabular}{lllll}
\hline Type & $\partial T / \partial \log g$ & $\partial T / \partial[\mathrm{Fe} / \mathrm{H}]$ & $\Delta \log g$ & $\Delta[\mathrm{Fe} / \mathrm{H}]$ \\
\hline G8 & 150 & 258 & 0.46 & 0.27 \\
K0 & 200 & 232 & 0.52 & 0.45 \\
K1-2 & 203 & 245 & 0.68 & 0.56 \\
K3-4 & 168 & 277 & 0.79 & 0.48 \\
\hline
\end{tabular}

The lack of large enough samples of stars with homogeneously determined metal abundances makes it difficult to extend this analysis to stars of other spectral types although it is observed that G-K subgiants behave similarly in the abundance-temperature plane if one includes those analysed by Glebocki (1972) with those from the narrow-band work.

\section{Conclusion}

The sensitivity to gravity and metal abundance of the relation between temperature and spectral types of G8 to $\mathrm{K} 4$ giants has been investigated, indicating that a factor of three in the gravity or two in the metal abundance affects the relation by up to $100 \mathrm{~K}$ or about one spectral sub-type. 


\section{References}

Gebocki, R.: 1972, Acta Astron. 22, 141.

Johnson, H. L. and Morgan, W. W.: 1953, Astrophys. J. 117, 313.

Morgan, W. W.: 1937, Astrophys J. 85, 380.

Pannekoek, A.: 1922, Bull. Astron. Inst. Neth. 1, 107.

Roman, N. G.: 1952, Astrophys. J. 116, 122.

Williams, P. M.: 1971, Monthly Notices Roy. Astron. Soc. 153, 171.

Williams, P. M.: 1972, Monthly Notices Roy. Astron. Soc. 158, 361.

\section{DISCUSSION}

Bidelman: What were the sources of your spectral types? Williams: Preferably from Johnson and Morgan, otherwise from Roman. 